\title{
Research on Different coupling methods in Jack-up Simulation
}

\author{
Yonggang Cui ${ }^{1,}$ a, Xiaojian $\mathrm{Pi}^{2,}$ b , Shilun Feng ${ }^{1, b}$ *, Jingjing $\mathrm{Li}^{1, \mathrm{c}}$, Chen Bian ${ }^{1, \mathrm{c}}$ \\ 1. Institute of Civil Engineering, Tianjin University, Tianjin 300072, China \\ 2. China Southwest Architecture Design and Research Institute Corporation, Chengdu 610084, \\ acui199215@163.com, ${ }^{b}$ shilunfeng@126.com, ${ }^{c}$ lijingjingouc@163.com,
}

Keywords: Jack-up, Coupling, Rack and Pinion, Finite element.

Abstract. Jack-up platform occupies an important position in marine oil exploitation due to its advantages. A finite element model of a Jack-up was constructed and analyzed. And Different coupling methods were used to simulate the links between the boat hull and legs. The two kinds of coupling methods are the coupling DOFs/Mstr method and the Rigid Region coupling method. And the calculation results showed that the Rigid Region coupling method was better than the coupling DOFs/Mstr method. The Rigid Region coupling method was recommended in the actual engineering.

\section{Introduction}

Jack-up Platform is widely used in marine oil exploitation because of its plenty advantages such as the construction technology is easy to grasp, the range of motion is large and the cost is low. However, due to the complication of marine environment, the structure must be sufficiently analyzed to guarantee the reliability[1-4]. The finite element method is commonly used in engineering to create a finite element model and carry out static analysis for Jack-up platforms[5-7]. In order to simplify the finite element model and the calculation process, some local structures can be neglected in hull simulation. Among these local structures, Rack and Pinion, top guide and bottom guide can be replaced by couplings to connect the hull structure and the legs.

An finite element model for a Jack-up Platform is created by ANSYS in this paper, and two kinds of coupling methods including the coupling DOFs/Mstr method and the Rigid Region coupling method are both used to simulate rack and pinion, top guide and bottom guide to stimulate the link between the boat hull structure and legs.

\section{Finite Element Model for Jack-up}

The hull structure of the Platform used in this paper is triangular in shape, and the platform has truss framed legs[8]. The finite element model is created by the ANSYS software, and the plate structure and beam structure is simulated by the Shell 181 and the Beam 188, respectively. The hull structure is shown in Fig. 1. The origin of this model is at the sea level. The direction of $\mathrm{z}$ axis is sticking straight up. The direction of $\mathrm{x}$ begins from the stern and along the symmetry axis of the platform, and the direction of $y$ begins from the symmetry axis of the platform and along the stern, as is shown in Fig. 1. The degree of freedom(DOF) in different directions are coupled for rack and pinion, top guide and bottom guide. All DOFs in X,Y,Z direction are coupled between the legs and the hull to simulate rack and pinion, while the DOFs in $\mathrm{X}, \mathrm{Y}$ direction are coupled between the nodes at guide areas and their corresponding nodes on the legs to simulate the links between the legs and the guides. The coupling DOFs/Mstr method and the Rigid Region coupling method are used in these coupling, respectively, as is shown in Fig. 2. Hinge constraint is used at the bottom of the model legs, as is shown in Fig. 3. Therefore we get two models which only differ in the type of coupling. 


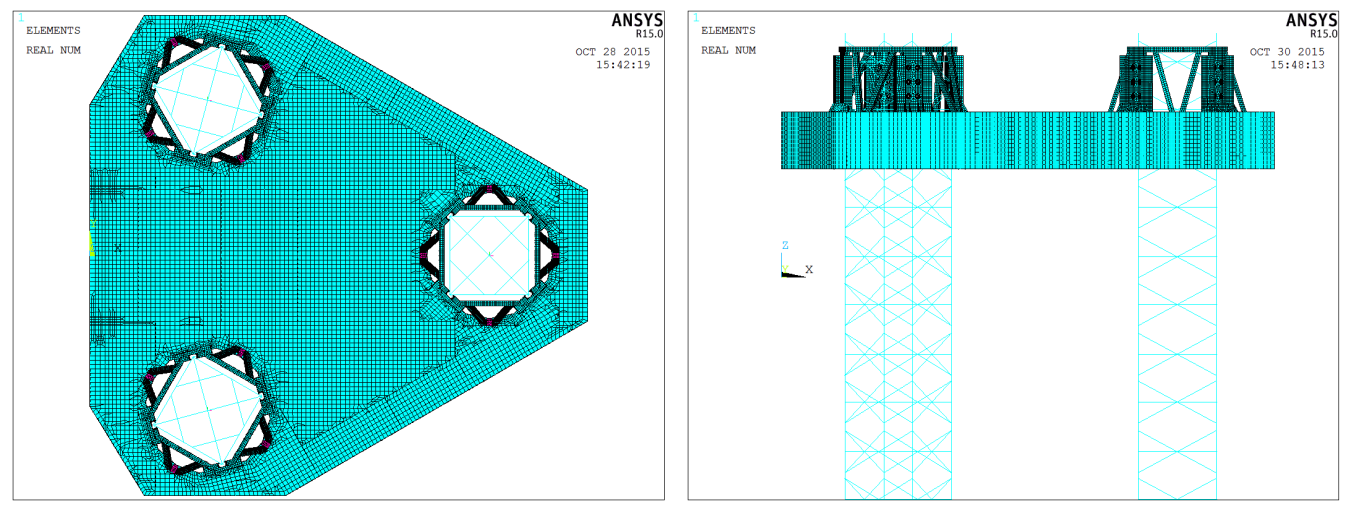

Fig. 1 Finite element model of Jack-up

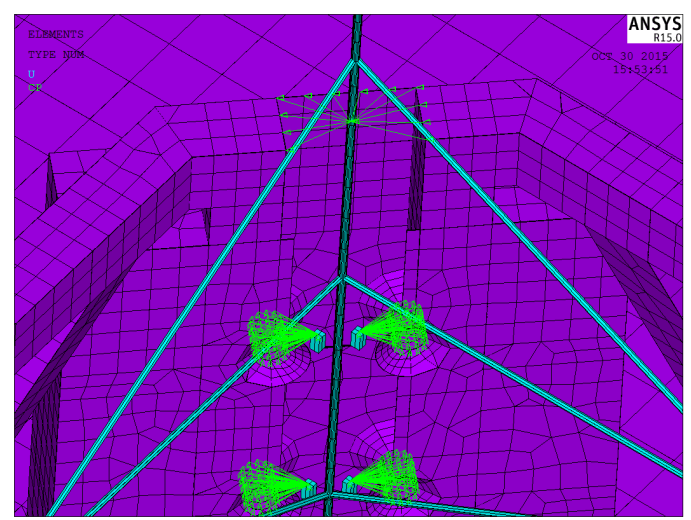

a Cupl DOFs/Mstr coupling

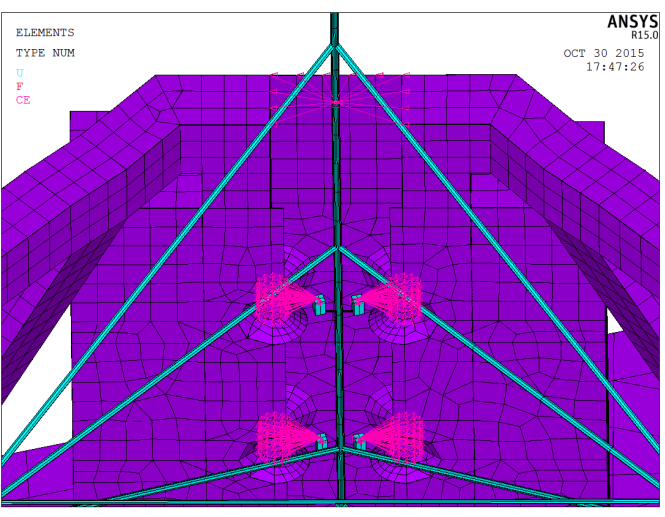

b Rigid Region coupling

Fig. 2 the coupling DOFs/Mstr method and the Rigid Region coupling method

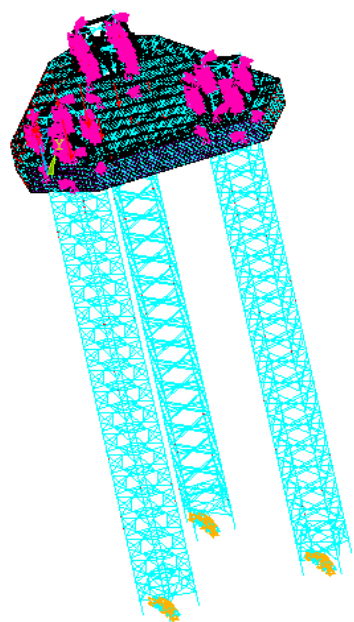

Fig. 3 the constraint of the jack-up model

\section{Loads Application}

As one of the most important parts of Jack-up, the cantilever is stretched out of the hull structure during drilling condition. The cantilever loads must be applied to ensure that the gravity center of the cantilever is identical to the actual engineering condition. According to the jack-up operating manual, the cantilever load is $35387.265 \mathrm{kN}$, and its coordinate value should be $(-14.52,0.477)$. Loads of cantilever are distributed to four real support locations: A,B,C and D, as is shown in Table 1. In Table 1, the negative value denotes the direction of this force is contrary to $\mathrm{Z}$ axis. 
Table 1 values of different cantilever load locations

\begin{tabular}{cccccc}
\hline Location & $\mathrm{A}$ & $\mathrm{B}$ & $\mathrm{C}$ & $\mathrm{D}$ & total \\
\hline $\mathrm{X}[\mathrm{m}]$ & 0.5747 & 14.6304 & 0.5747 & 14.6304 & \\
\hline $\mathrm{Y}[\mathrm{m}]$ & 9.144 & 9.144 & -9.144 & -9.144 & \\
\hline $\begin{array}{c}\text { Value of } \\
\text { Force[kN] }\end{array}$ & -36628.547 & 18012.513 & -35706.146 & 18934.915 & -35387.265 \\
\hline
\end{tabular}

The loads of cantilever are applied in the two models which differ in the coupling DOFs/Mstr method and the Rigid Region coupling method. And the other loads in the platform are assumed to be zero to simplify the problem. The Eq. 1 and formula Eq. 2 is used to calculate the gravity center of the model according the reaction force of legs.

$$
\begin{aligned}
& x=\frac{F_{1} * x_{1}+F_{2} * x_{2}+F_{3} * x_{3}}{F_{1}+F_{2}+F_{3}} \\
& y=\frac{F_{1} * y_{1}+F_{2} * y_{2}+F_{3} * y_{3}}{F_{1}+F_{2}+F_{3}}
\end{aligned}
$$

Where $\mathrm{x}$ denotes the $\mathrm{x}$-coordinate of the platform gravity center; $F_{1}, F_{2}, F_{3}$ denotes the reaction force of three legs, respectively; $x_{1}, x_{2}, x_{3}$ denotes the x-coordinate of the reaction force of three legs, respectively. Where y denotes the of the platform gravity center; $y_{1}, y_{2}, y_{3}$ denotes the $\mathrm{y}$-coordinate of the reaction force of three legs, respectively.

\section{Calculation Results}

The calculation results for the platform gravity center are shown in Table 2 and Table 3, and the Mises Stress of hull is shown in Fig. 2. The x-coordinate of the platform gravity center should be -14.52 , and y-coordinate should be 0.477. Comparing the results in Table 2 and Table 3, the

Table 2 Calculation results of gravity center x-coordinate

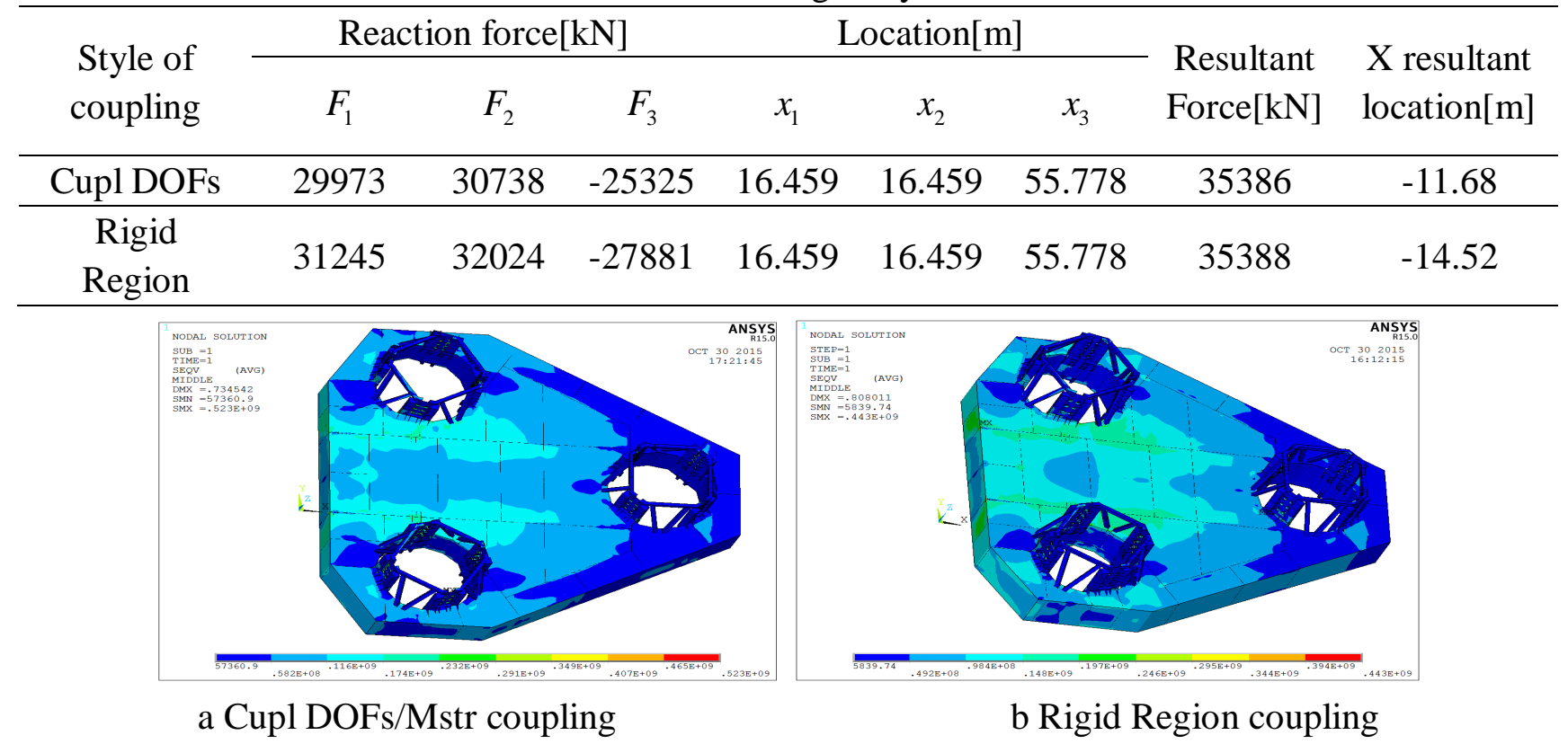

Fig. 4 Mises Stress of the boat hull 
Rigid Region coupling method is obviously more closer to the theoretical one than the coupling DOFs/Mstr method, while coupling by Cupl DOFs/Mstr has bigger difference with theoretical one than the Rigid Region coupling.

Table 3 Calculation results of gravity center y-coordinate

\begin{tabular}{|c|c|c|c|c|c|c|c|c|}
\hline \multirow{2}{*}{$\begin{array}{l}\text { Style of } \\
\text { coupling }\end{array}$} & \multicolumn{3}{|c|}{ Reaction force $[\mathrm{kN}]$} & \multicolumn{3}{|c|}{ Location $[\mathrm{m}]$} & \multirow{2}{*}{$\begin{array}{l}\text { Resultant } \\
\text { Force }[\mathrm{kN}]\end{array}$} & \multirow{2}{*}{$\begin{array}{l}\text { Y resultant } \\
\text { location }[\mathrm{m}]\end{array}$} \\
\hline & $F_{1}$ & $F_{2}$ & $F_{3}$ & $\mathrm{y}_{1}$ & $\mathrm{y}_{2}$ & $\mathrm{y}_{3}$ & & \\
\hline Cupl DOFs & 29973 & 30738 & -25325 & -21.641 & 21.641 & 0 & 35386 & 0.47 \\
\hline Rigid Region & 31245 & 32024 & -27881 & -21.641 & 21.641 & 0 & 35388 & 0.48 \\
\hline
\end{tabular}

The maximum Mises Stress of hull is $523 \mathrm{MPa}$ and $443 \mathrm{MPa}$, respectively. It shows that the Cupl DOFs/Mstr method induces stress concentration in the local structure. So the Cupl DOFs/Mstr method should be used carefully.

\section{Conclusion}

In this paper we create two finite element models for a Jack-up Platform using different kinds of coupling. By comparing the calculation results, we can know that: (1) the Rigid Region method is much more close to the theoretical value and is better than the coupling DOFs/Mstr method; (2) the maximum Mises Stress of the boat hull of two models differs much because the model using Cupl DOFs/Mstr method induces stress concentration. Generally, the rigid Region coupling is recommended in the Jack-up platform finite element model to simulate the link between the hull and legs.

\section{Acknowledgements}

The research was financially supported by National Science Foundation of China(51239008), National Science Foundation of China(51379145), National Key Basic Research Program of China (2014CB046800) and Ministry of Transport of the People's Republic of China (20130318740050).

\section{References}

[1] Cassidy M.J., et al: Evaluation of long-term extreme response statistics of jack-up platforms, Ocean Engineering, Vol. 29(2002), pp. 1603-1631.

[2] Hambly E. C., Imm G. R., Stahl B: Jackup Performance and Foundation Fixity Under Developing Storm Contions, OTC 6466, 1990.

[3] Mao Dongfeng, Zhang Minghui, Zhang Laibin, et al: Sliding risk of jack-up platform re-installation close to existing footprint and its countermeasure, Petroleum Exploration and Development, Vol. 42(2015), pp.259-264.

[4] Zhang Youhu, Britta Bienen, Mark J. Cassidy: Jack-up push-over analyses featuring a new force resultant model for spudcans in soft clay, Ocean Engineering, Vol. 81(1)(2014)pp. 139-149.

[5] Y.G. Cao, S.H. Zhang: Failure analysis of a pinion of the jacking system of a jack-up platform, Engineering Failure Analysis, Vol. 33(2013), pp. 212-221.

[6] Zhang Puyang, Yu Xiaoyang, Ding Hongyan: Spudcan bearing capacity calculation of the offshore jack-up drilling platform during the preloading process, Petroleum Exploration and Development, Vol. 38(5)(2011),pp. 613-619.

[7] Jiao Yang, Feng S L, Liu H Y: A quick method for the stability of jack-up platform towage, 2014 International Conference on Civil Engineering, Energy and Environment (CEEE 2014), Hong Kong,13-14 December 2014, pp. 119-124.

[8] Pederson P. Optimal joint positions for space trusses, J.Struct.Div.ASCE. 1973, 99(ST10). 